\title{
Magnetospheric conditions near the equatorial footpoints of proton isotropy boundaries
}

\author{
V. A. Sergeev ${ }^{1}$, I. A. Chernyaev ${ }^{1}$, V Angelopoulos ${ }^{2}$, and N. Y. Ganushkina ${ }^{3,4}$ \\ ${ }^{1}$ St. Petersburg State University, Ulyanovskaya 1, 198504 St. Petersburg, Russia \\ ${ }^{2}$ Department of Earth, Planetary and Space Sciences and Institute of Geophysics and Planetary Physics, \\ University of California, Los Angeles, USA \\ ${ }^{3}$ Department of Atmospheric, Oceanic and Space Sciences, University of Michigan, Ann Arbor MI, USA \\ ${ }^{4}$ Finnish Meteorological Institute, Helsinki, Finland \\ Correspondence to: V. A. Sergeev (victor@geo.phys.spbu.ru)
}

Received: 1 September 2015 - Revised: 13 November 2015 - Accepted: 26 November 2015 - Published: 9 December 2015

\begin{abstract}
Data from a cluster of three THEMIS (Time History of Events and Macroscale Interactions during Substorms) spacecraft during February-March 2009 frequently provide an opportunity to construct local data-adaptive magnetospheric models, which are suitable for the accurate mapping along the magnetic field lines at distances of 6-9 Re in the nightside magnetosphere. This allows us to map the isotropy boundaries (IBs) of 30 and $80 \mathrm{keV}$ protons observed by low-altitude NOAA POES (Polar Orbiting Environmental Satellites) to the equatorial magnetosphere (to find the projected isotropy boundary, PIB) and study the magnetospheric conditions, particularly to evaluate the ratio $K_{\mathrm{IB}}\left(R_{\mathrm{c}} / r_{\mathrm{c}}\right.$; the magnetic field curvature radius to the particle gyroradius) in the neutral sheet at that point. Special care is taken to control the factors which influence the accuracy of the adaptive models and mapping. Data indicate that better accuracy of an adaptive model is achieved when the PIB distance from the closest spacecraft is as small as 1-2 Re. For this group of most accurate predictions, the spread of $K_{\mathrm{IB}}$ values is still large (from 4 to 32 ), with the median value $K_{\mathrm{IB}} \sim 13$ being larger than the critical value $K_{\mathrm{cr}} \sim 8$ expected at the inner boundary of nonadiabatic angular scattering in the current sheet. It appears that two different mechanisms may contribute to form the isotropy boundary. The group with $K \sim[4,12]$ is most likely formed by current sheet scattering, whereas the group having $K_{\mathrm{IB}} \sim[12,32]$ could be formed by the resonant scattering of low-energy protons by the electromagnetic ion-cyclotron (EMIC) waves. The energy dependence of the upper $K$ limit and close proximity of the latter event to the plasmapause locations support this conclusion.
\end{abstract}

We also discuss other reasons why the $K \sim 8$ criterion for isotropization may fail to work, as well as a possible relationship between the two scattering mechanisms.

Keywords. Magnetospheric physics (energetic particles precipitating; magnetospheric configuration and dynamics)

\section{Introduction}

Adiabatic motion of charged particles in the trap geometry of the geomagnetic field conserves the empty atmospheric loss cone in the particle distributions. However, in the regions where the particle gyroradius $\rho$ becomes comparable to the magnetic curvature radius $\left(R_{\mathrm{c}}\right)$ and adiabatic approximation is violated, the pitch-angle scattering fills the loss cone and leads to particle precipitation into the ionosphere. For the protons with energies ranging between a few tens and $100 \mathrm{keV}$, the boundary between adiabatic and nonadiabatic particle motion occurs near the center of the tail current sheet on the nightside at $r \sim 6-9 \operatorname{Re}$ (e.g., Sergeev and Tsyganenko, 1982; Shevchenko et al., 2010; Wang et al., 2012; Yue et al., 2014). Tailward of that boundary a strong current sheet scattering (CSS) provides the nearly isotropic proton angular distributions in the tail plasma sheet (e.g., Ganushkina et al., 2005; Yue et al., 2014) whose precipitation forms an extended isotropic proton precipitation region, the proton auroral oval (Sergeev et al., 1983; Donovan et al., 2003; Meurant et al., 2007). The equatorward boundary at which the ratio of precipitated to trapped fluxes quickly drops down below 1 (called the isotropy boundary, IB) is usually sharply de- 
fined and well-observed at any particular energy. It provides an important low-altitude marker of the boundary between adiabatic and nonadiabatic motion in the equatorial current sheet for the particle population.

The normal $\left(B_{z}\right)$ and radial $\left(B_{r}\right)$ magnetic field components and their gradients usually control the angular scattering amplitude in the tail current sheet. In the tail-like geometry the rough criterion separating full loss cone coverage (strong scattering) from partial (incomplete) cone filling conditions for this mechanism is approximately

$K_{\mathrm{cr}}=R_{\mathrm{c}} / \rho \approx B_{z}^{2} /\left(\mathrm{d} B_{r} / \mathrm{d} z \cdot G\right) \cong 8$,

where $G=m V / e$ is the particle rigidity. This value $K_{\mathrm{cr}}=8$ was previously obtained using particle tracing in the simple 1-d current sheet models (e.g., Sergeev and Tsyganenko, 1982; Delcourt et al., 1996) or using a superposed 1-d current sheet and dipole field (Sergeev and Malkov, 1988); up to now the criterion (1) has been used in the majority of papers addressing the CSS-based isotropy boundaries (e.g., Shevchenko et al., 2010; Yue et al., 2014; Liang et al., 2014). The uncertainty regarding this numerical criterion and its dependence on the $B$-field parameters (e.g., distance etc) have not been systematically investigated.

The observational test of this relationship is an important task, which is difficult to perform because the isotropy boundary is formed in the equatorial magnetotail where the filling of the loss cone is hard to observe due to the small size of the loss cone (about $1^{\circ}$ ). At the same time, the IB is robustly and easily observed at low altitudes, far from its formation place. Therefore, to test the conditions at the IB footpoints in the current sheet, we need nearly conjugate observations in both regions, reliable magnetic field line tracing between them as well as a way to control the accuracy of this mapping. The two latter requirements are very difficult to implement. Previous works comparing magnetospheric and ionospheric observations were mostly based on statistical empirical Tsyganenko models (Tsyganenko, 1995) or average-pressure-based models (e.g., Yue et al., 2014) used to find the isotropy boundary location in the neutral sheet according to Eq. (1). Then this location was mapped to the ionosphere, to compare predictions with the IB observations at low altitudes. Such comparisons typically confirm similarities in their local time, solar wind and activity dependencies, the predicted and observed IB latitudes agreed on average to within $1-2^{\circ}$ CGLat. The standard deviations were also comparable to that value (see, e.g., Sergeev and Gvozdevsky, 1995; Shevchenko et al., 2010; Yue et al., 2014), which characterizes the typical mapping uncertainty of statistical models (Nishimura et al., 2011). Therefore, observational confirmation of the CSS mechanism and validation of its numerical criterion (of Eq. 1) still awaits a better model and a better control of the mapping uncertainty.

Recently some evidence has been published that another mechanism - pitch-angle scattering by the electromagnetic ion-cyclotron (EMIC) plasma waves - can act in the flux tubes adjacent to the isotropy boundary. An association between EMIC waves and proton precipitation (often isotropic) is easier to establish for the detached precipitation structures (Yahnin and Yahnina, 2007) than for the isotropy boundary case. Liang et al. (2014) analyzed numerous cases of the inverse proton energy dispersion of low-energy (1-20 keV) protons, provided their theoretical explanation, and showed a couple of cases in which EMIC waves were directly observed in the equatorial magnetosphere in the sector where the inverse IB dispersion was identified. Sergeev et al. (2015) presented a statistical survey of the IB morphology on the nightside. They identified a few morphological features (such as frequent occurrence of coincident IBs in 30 and $80 \mathrm{keV}$ proton energy channels, frequent multiple dropouts of precipitated to trapped flux ratio near the IB location, and observations of newly emerging isotropic precipitation equatorward of the previous IB) which are inconsistent with a simple CSS-based model but can be explained in terms of a wave-particle interaction mechanism. These results put a question mark over the roles of the CSS and wave mechanisms in forming the proton isotropy boundary.

An obvious way to address these problems and to test the $K$ value in the magnetospheric projection of the isotropy boundary is to use the data-adapted magnetospheric modeling in which several free model parameters are tuned to achieve the best possible agreement between the modeled magnetic field and the spacecraft magnetic field observations (e.g., Kubyshkina et al., 2011). The advantage of such models is that the model predictions are based on real observations, and they also allow us to perform some model quality estimates. Their difficulty is that the amount of data for the modeling (the number of the spacecraft which measure the magnetic field) is usually small and the results are very sensitive to the spacecraft coverage. In this paper we test the adaptive modeling approach by using the unique possibility of THEMIS (Time History of Events and Macroscale Interactions during Substorms) orbits during the 2009 tail season, in which a cluster of three spacecraft frequently occurred in the equatorial region in the vicinity of the field line connected to the low-altitude NOAA POES (Polar Orbiting Environmental Satellites), which at that time observed the isotropy boundary. We construct adaptive models for these events, analyze the $K$ values in about 50 such conjunctions and discuss the implications concerning the mechanism which is responsible for the formation of the proton isotropy boundaries.

\section{Observations}

Observations of trapped $\left(J_{90}\right)$ and precipitating $\left(J_{0}\right)$ energetic proton fluxes by the Space Environment Monitor-2 (SEM2) onboard the low-altitude $(\sim 850 \mathrm{~km})$ NOAA POES were available from http://ngdc.noaa.gov/stp/satellite/poes/. Here we use the low-energy Medium Energy Proton and Electron Detector (MEPED) proton channels (nominally $30-80 \mathrm{keV}$ 


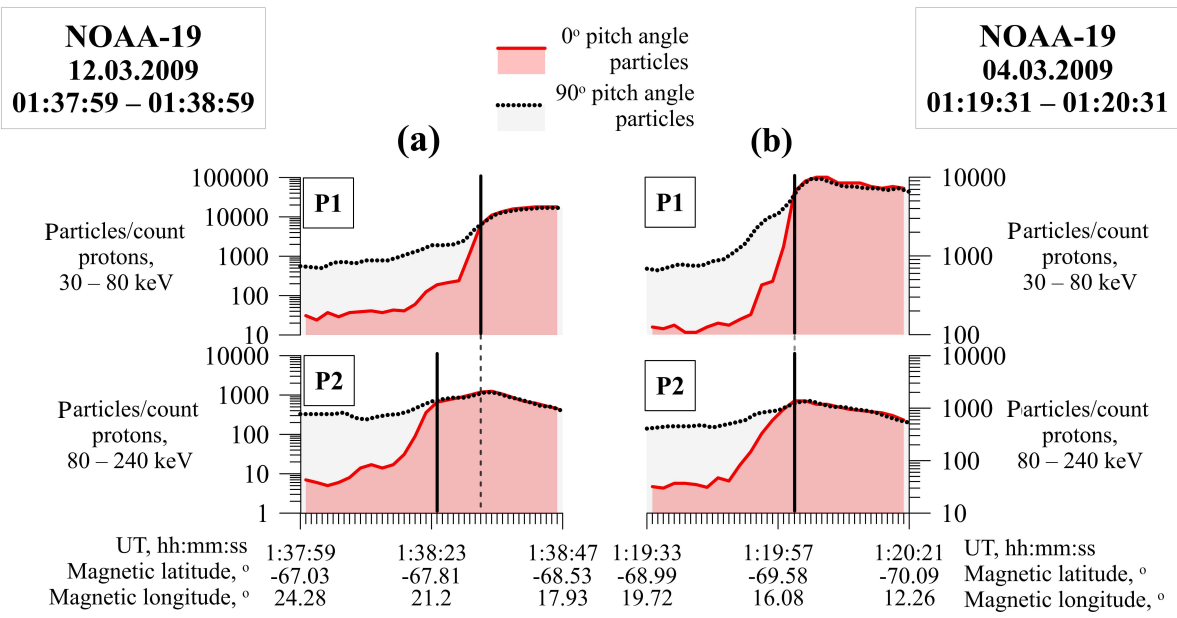

Figure 1. Count rates of trapped ( $J_{90}$, black dotted line) and precipitating ( $J_{0}$, red line) energetic ion fluxes provided by NOAA-19 in premidnight sector. Isotropy boundaries are marked by vertical lines. Event (a) corresponds to the standard energy dispersion and event (b) to the "no IB dispersion" case.

(P1) and $80-240 \mathrm{keV}(\mathrm{P} 2)$ at $2 \mathrm{~s}$ time resolution; Evans and Greer, 2004). Figure 1 provides two examples of isotropic fluxes $\left(J_{0} \sim J_{90}\right)$ seen on the poleward (right) side of each panel but with a (grey shaded) highly anisotropic $\left(J_{0} \gg J_{90}\right)$ flux region on the equatorial side (left side of each panel). The boundary between these two regions, namely the last isotropic flux measurement before the sharp drop of precipitated flux on the low-latitude side of the isotropic zone, is usually well defined, and it is the isotropy boundary (IB) which is the focus of our study. The current sheet scattering (CSS) mechanism predicts that higher-energy protons should have their adiabatic-to-nonadiabatic scattering boundary closer to the Earth than lower-energy protons. In other words, the $80 \mathrm{keV}$ proton IB should be observed at a lower latitude, compared to the $30 \mathrm{keV}$ proton IB. Such energy-dependent IB displacement is seen in event (a), but virtually no dispersion is observed in event (b). According to a recent NOAA-POES-based survey of the IB energy dispersion patterns presented by Sergeev et al. (2015), these two categories are most frequently observed in the nightside auroral oval. Due to the radiation-related detector degradation with time, the lower energies of $30 / 80 \mathrm{keV}$ proton channels evolve with time; below we use their corrected values for March 2009 according to the Asikainen et al. (2012) results. Specifically, for a different NOAA-type satellite the corresponding corrected energies were taken to be $30 / 80 \mathrm{keV}$ (NOAA-19), 30/94 keV (NOAA-18), 36/92 keV (METOP2) and $46 / 122 \mathrm{keV}$ (NOAA-17). (Here we note that the corrected energy thresholds are slightly different for precipitated and trapped particles at every spacecraft and that improved correction factors have been recently published by Sadanger et al. (2015). However, these differences do not seriously affect any of the results in our paper.)
Previous modeling studies by Lvova et al. (2005) and Shevchenko et al. (2010) identified that, for the wide range of disturbed-to-quiet activity conditions, the proton IBs in the equatorial nightside magnetosphere (due to the CSS mechanism) should be located at geocentric distances of 5-9 Re. Ideally, to ensure accurate mapping, magnetospheric observations should be available at a few locations covering this distance range in the conjugate equatorial magnetosphere. This requirement was frequently satisfied for the group of three THEMIS spacecraft in February-March 2009. During that time ThA, ThD and ThE moved closely to each other along similar near-equatorial orbits, all having a $\sim 24 \mathrm{~h}$ orbital period and the apogee of $\sim 12 \mathrm{Re}$. As illustrated in Fig. 2, during one orbit three spacecraft crossed this distance range of interest twice (on the outbound and inbound portions of each orbit) at distances of 1 to $4 \mathrm{Re}$. The next step was to identify those NOAA spacecraft auroral zone crossings which are magnetically conjugate to THEMIS in the region of interest. If the time difference between THEMIS and NOAA exceeded $3 \mathrm{~h}$, the crossing was removed from further analysis.

In the next step, adaptive modeling was performed (see below) to refine the conjunction and to perform the accurate mapping of the observed isotropy boundary from the NOAA altitude $(\sim 850 \mathrm{~km})$ to the neutral sheet region of the equatorial magnetosphere, namely to the field line point where the radial magnetic field component changes its sign. The location of this projected IB (PIB in Fig. 2) was then compared to the location of the closest THEMIS spacecraft, and the points whose distance exceeded 6 Re were discarded. After this procedure the list of 50 conjunctions which occurred between 24 February and 27 March in the year 2009 was obtained for detailed study; the conjunctions are shown in Fig. 2b. Selected crossings are located in the nightside MLT sector clustering 
at 21-23 and 01-03 h MLT. A gap near midnight is due to the combination of a low occurrence of NOAA crossings in that MLT sector and the necessity to discard the conjunction when any of three THEMIS spacecraft was in the Earth shadow.

A typical configuration of three THEMIS spacecraft on the outbound portion of their orbit is illustrated in Fig. 2a. Here the ThD leads the group, being separated by 3-4 Re from the ThE-ThA pair; this provides the information about the radial gradient of the magnetic field. In the pair, the ThE spacecraft is $\sim 1$ Re above ThA, providing information about the vertical $B$ gradients, that is about the current density in the equatorial current sheet. This information is invaluable for the accurate evaluation of the CSS-related isotropy boundary (see below). As for the dawnside crossings, they follow in the same order, but ThD is now at the smallest geocentric distance in the group. A peculiarity of the inbound orbits is that, besides the three near-Earth THEMIS spacecraft, the other two THEMIS spacecraft (ThB and ThC, which have the apogees in the middle tail) occasionally crossed the inner magnetosphere inwards simultaneously with other spacecraft, so in a few events the data of four to five spacecraft were also available for the modeling.

The generation of the EMIC waves and the wave scattering efficiency are known to be sensitive to the cold plasma distribution, and the associated detached proton precipitation regions are known to be observed near the plasmapause location (Yahnin and Yahnina, 2007). To characterize the plasmapause position we looked through the variation in the spacecraft potential along the THEMIS trajectories. Strong (and often sharp) potential variation (roughly between 5 and $10 \mathrm{~V}$, characteristic of large plasma density changes) was regarded as the plasmapause signature. The plasmapause observation closest in time to (usually within $2 \mathrm{~h}$ of) the isotropy boundary observations was taken as the plasmapause location in our data set.

\section{Adaptive models and control of the model accuracy}

The adaptive modeling approach uses the magnetospheric model, which describes different current systems. In each particular event (at any specific time during the event), several free model parameters are varied to fit the magnetic fields, which are observed simultaneously by a few (several) magnetospheric spacecraft. In the case of good spacecraft coverage in a localized magnetospheric region of interest (like in our case), this method provides the model configuration for any specific time which is expected to represent the real configuration in this localized domain as closely as possible (in our case - in the near-equatorial region in the vicinity of the mapped isotropy boundary). In our study we use the AM03 version of the adaptive model previously described by Kubyshkina et al. $(2009,2011)$. The formulation for the model current systems is borrowed from the T96 model (Tsy- ganenko, 1995) with a couple of additions. That is to say, the AM03 model includes the additional embedded thin current sheet and a possibility to vary the current sheet tilt in the tail. The initial fit is obtained by varying the input model parameters (solar wind flow pressure, IMF $B_{z}$, Dst, treated as free parameters) and intensities of all model current systems. The following step is to include the additional thin current sheet and vary its parameters (sheet thickness and intensity) to improve the fit (see more details in Kubyshkina et al., 2011). In the trial-and-search procedure, the least square minimization solution is looked for for the fit function

$\delta B_{\mathrm{AM} 03}=\left(\Sigma_{i j}\left(B_{i j}^{\mathrm{mod}}-B_{i j}^{\mathrm{obs}}\right)^{2} / N\right)^{1 / 2}$,

where $B_{i j}$ is the $j$ th $B$ component at the $i$ th (of $N$ ) spacecraft. This parameter can also characterize the accuracy of the fit solution.

After specifying the magnetic field model for the time of the IB measurement and after performing the field line mapping of the IB magnetic field line to the equatorial magnetosphere until its crossing with the neutral sheet surface (point PIB in Fig. 2a), the value of the $K$ parameter was computed at this neutral sheet point as

$K=e B R_{\mathrm{c}} /\left(2 m_{p} E\right)^{1 / 2}$,

where $E$ is the proton energy and the curvature radius $R_{\mathrm{c}}$ is numerically evaluated at this point using the coordinates of the traced field line. We emphasize that in the simple model with a central current sheet the $R_{\mathrm{c}}$ value is controlled by both the $B_{z}$ component value in the neutral sheet and the current sheet density $\left(R_{\mathrm{c}}=B_{z} /\left(d B_{r} / d z\right)\right.$ in the case of zero dipole tilt), so a control of the $Z$ gradient using a pair of vertically separated ThA and ThE spacecraft is a necessary condition for the accurate evaluation of the $K$ value. How closely the model represents this gradient can be evaluated by computing the difference.

$$
\begin{aligned}
\delta B_{X}^{\mathrm{CS}} & =\mid\left(B_{X}^{(\mathrm{ThE}, \mathrm{obs})}-B_{X}^{(\mathrm{ThA}, \mathrm{obs})}\right)-\left(B_{X}^{(\mathrm{ThE}, \mathrm{mod})}\right. \\
& \left.-B_{X}^{(\text {ThA, mod })}\right) \mid
\end{aligned}
$$

where the indices obs (or mod) denote the $B_{x}$ component values observed (or modeled) at the corresponding spacecraft locations.

For the equatorial current sheet the T96 and AM03 models exploit the smooth functions which describe a simple equatorial Harris-like current sheet with a peak current being in the current sheet central plane. During the growth phase this picture should be modified by the growth of the embedded thin current sheet (see, e.g., Petrukovich et al., 2011). This feature can be represented by the AM03 model, which includes the possibility of an embedded current sheet with variable thickness (e.g., Kubyshkina et al., 2011). However, during the local dipolarizations and other substorm-related (or flowburst-related) perturbations which disturb a smooth configuration, the quality of the adaptive model degrades. To control these effects quantitatively, we computed the detrended 

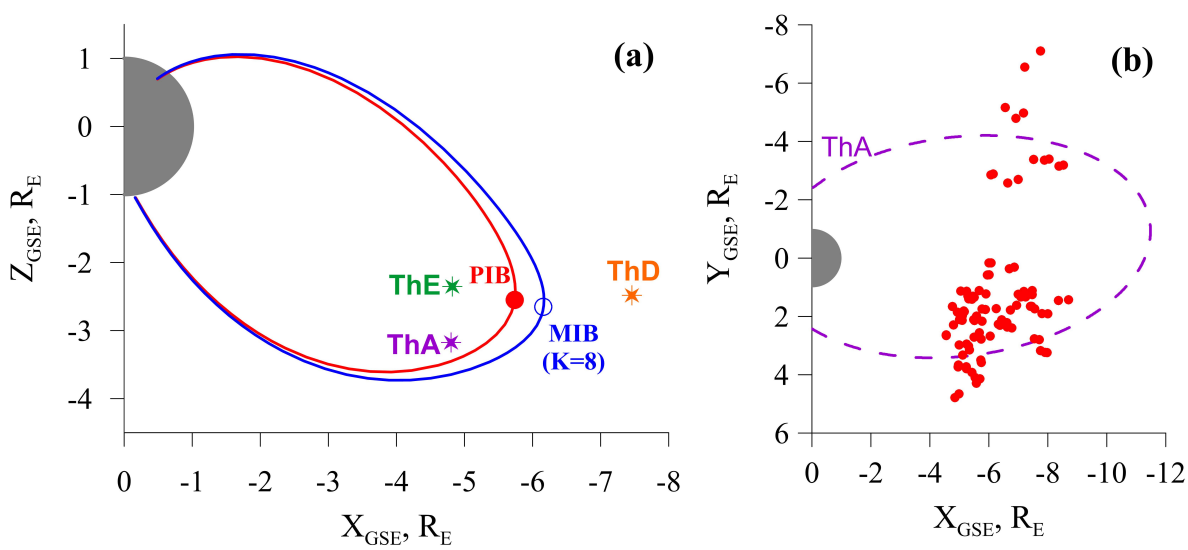

Figure 2. Panel (a): $X Z$ projections of the magnetic field line starting from the observed isotropy boundary (red; labeled PIB) and of the field line crossing the critical equatorial point (where $K=8$; blue; labeled MIB), together with locations of three THEMIS spacecraft. Panel (b): equatorial mapping of sample ThA trajectory on 4 March 2009 (dashed line) and the locations of projected isotropy boundaries (PIBs) in the final selected conjunction events.

$B$-field standard deviation $\delta B^{\text {obs }}$ averaged over the $10 \mathrm{~min}$ time interval centered on the time of the IB observation. Furthermore, we visually examined the THEMIS $B$-field time series and removed a few cases showing the dipolarization fronts and injection features during this 10 min time interval. We also removed a couple of events in which the $\mathrm{AE}$ (auroral electrojet) index exceeded $250 \mathrm{nT}$ and demonstrated substorm signatures. Finally, based on the $B$-field variability index, we excluded a few events in which $\delta B_{\mathrm{AM} 03}$ exceeded $10 \mathrm{nT}$.

For our purpose, a useful measure to characterize the coverage can be the distance between the projected IB point and the THEMIS spacecraft closest to it. We tried two such measures, one characterizing the difference between the geocentric distances of these two points (scalar difference $\Delta R_{1}$, which ignores azimuthal separation between two points) and another one characterizing the true distance between two points $\left(\Delta R_{2}\right)$. The events with $\delta R_{2}$ exceeding $6 \mathrm{Re}$ were discarded from the analysis.

Finally, after applying all the abovementioned selection procedures, we selected a list of 50 conjugate isotropy boundary crossings, each including two isotropy boundaries (at 30 and $80 \mathrm{keV}$ nominal energies; 100 data points available altogether). For these IB conjunctions a system of diagnostic parameters $\left(\delta B_{\mathrm{AM} 03}, \delta B^{\text {obs }}, \delta B_{x}^{\mathrm{CS}}, \Delta R_{1}, \Delta R_{2}\right)$ is available to characterize the quality of the adaptive modeling. The distribution of parameters for this data set is shown in Fig. 3. The data set is predominantly obtained during very quiet conditions ( $\mathrm{AE}<80 \mathrm{nT}$ for $3 / 4$ of data points, Fig. 3d), with the bulk of the isotropy boundaries staying at relatively high latitudes $65-68^{\circ}$ CGLat (Fig. 3a). The mapped IB distances nevertheless cover the expected range between 5 and $9 \mathrm{Re}$ (Fig. 3b). In addition to their normal values of 5 to $7 \mathrm{Re}$, The concurrent plasmapause positions (Fig. 3c) show a group of 12 events with unusually distant $(8-9 \mathrm{Re})$ plasmapause lo-

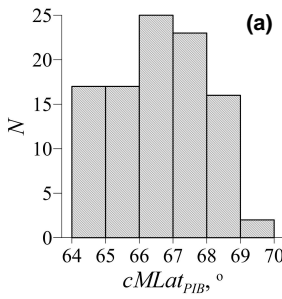

(a)

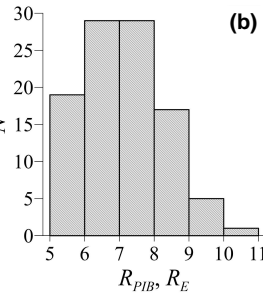

(b)
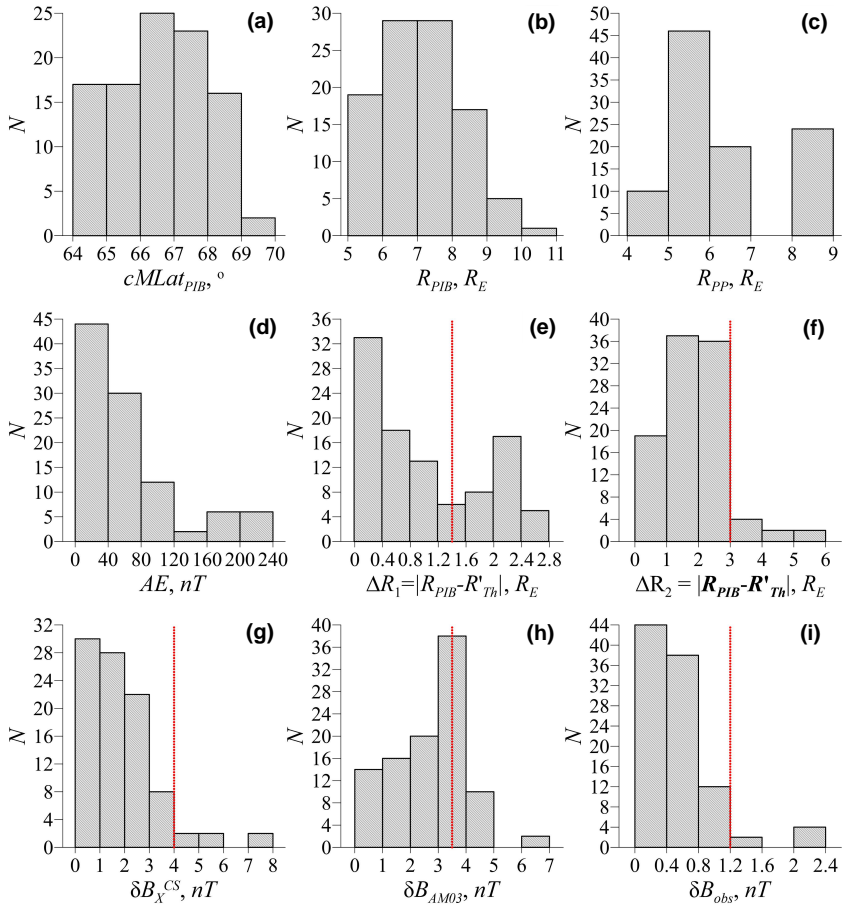

Figure 3. Parameter distributions characterizing our data set. (a) corrected magnetic latitudes (absolute values) of isotropy boundaries (30 and $80 \mathrm{keV}$ IBs are plotted together); (b) radial distances of projected IBs; (c) plasmapause positions (along THEMIS trajectories); (d) AE index; (e-f) distances between PIB and THEMIS spacecraft closest to this point; $(\mathbf{g}-\mathbf{i})$ parameters used to characterize the model accuracy (see text for explanations). The red vertical lines show the maximal parameter value allowed for the subgroup of the most accurate models (D1). 
cations. This is a specific feature of very quiet year 2009 . (The distant plasmapause cases are real, as is confirmed by the observations made by three THEMIS spacecraft which crossed this boundary at different times.)

A vast majority of the data points have low variability $\left(\delta B^{\text {obs }}<0.8 \mathrm{nT}\right.$, Fig. $\left.3 \mathrm{i}\right)$, a reasonably good fit to the spacecraft data $\left(\delta B_{\mathrm{AM} 03}<4 \mathrm{nT}\right.$, Fig. $\left.3 \mathrm{~h}\right)$ and a reasonably good approximation of the vertical $B_{x}$ component gradients $\left(\delta B_{x}^{\mathrm{CS}}<3 \mathrm{nT}\right.$, Fig. 3g). For the vast majority of selected events the coverage indicators are very good: the distance between isotropy boundary projection (PIB) and closest spacecraft is within $3 \mathrm{Re}$, with a big group of the IB crossings having $\Delta R_{1}<1 \mathrm{Re}$ and $\Delta R_{2}<2 \mathrm{Re}$.

Based on these quality indicators, after some investigation we divided the data set into two groups, predominantly based on the coverage indicators. In the most reliable mapping (group D1), we have 23 events, characterized by $\Delta R_{1}<$ $1.4 \mathrm{Re}, \Delta R_{2}<3 \mathrm{Re}, \delta B_{\mathrm{AM} 03}<3.5 \mathrm{nT}, \delta B^{\mathrm{obs}}<1.2 \mathrm{nT}$, and $\delta B_{x}^{\mathrm{CS}}<4 \mathrm{nT}$. In group D2 we have the remaining 27 events.

\section{Evaluation of $K$ parameters and local conditions in the vicinity of the isotropy boundary projection}

Using the relationship (3) the $K$ parameter values were evaluated in the intersection point of the neutral sheet and the magnetic field line, passing through the observed isotropy boundary (at the point PIB in Fig. 2a). A few important results are evident from Fig. 4, which shows the $K$ distribution. The first is that $K$ values show a large spread. In many cases the $K$ values are shifted considerably to high values compared to the $K=8$ value, predicted by the current sheet scattering (CSS) mechanism. Although the distribution has a peak at around $K=8$ (48 points within a factor of 2 from this value), the majority of data (about two thirds) have values exceeding the CSS threshold by a factor of 2 or more. This may have either physical reasons or be due to errors in the magnetic field model and, consequently, in the field line mapping. Another feature is that high $K$ values are more often seen in the lowest-energy channel P1 compared to P2.

To test how the quality of the models influences these results, we compare the $K$ distributions for two groups of events in Fig. 5. A more reliable group of models D1 (shown by red symbols) displays a compact $K$ distribution (ranging between 4 and 35), whereas the $K$ values between 35 and 67 are only observed in the group of less reliable models (D2; shown by black symbols). In our interpretation this means that the distance between modeled IB field line and closest observation point should not exceed 1.5-2 Re to guarantee good accuracy of the mapping in the near magnetosphere. The linear regression lines shown in Fig. 5a confirm quantitatively that deviation from $K=8$ toward high $K$ values increases for decreasing energy, being stronger than 17.1 and 12.7 for P1 and P2 channels, respectively (as indicated by blue crosses).

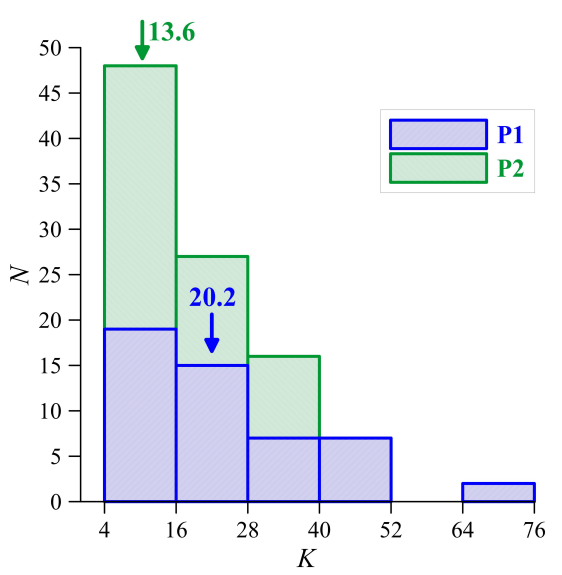

Figure 4. Distribution of the $K$-parameter values in the equatorial projection of the proton isotropy boundaries in the P1 (blue) and P2 (green) channels. The arrows show the median $K$ values in both channels as well as the $K=8$ critical threshold previously defined for the current sheet scattering mechanism.

In the group of the most accurate models the spread is still large enough, and on average the $K$ values still significantly exceed the critical number 8 . Suggesting that the $K$ values between 4 and 12 may be generated by the CSS mechanism, whereas the remaining large $K$ events can be due to some other reason, we investigated the differences between these two data sets and found one remarkable difference illustrated in Fig. 6. The more distant location of the low $K$ value boundaries is not surprising. Remarkably, there seems to be a systematic relationship between these points and plasmapause locations. The blue points (small $K$ values, possibly CSS mechanism) are systematically observed outside of the plasmapause, whereas a majority of the red triangles (large $K$ values) are observed within 1 Re from the plasmapause, and about one third of these points are seated inside the plasmasphere. Some scatter is expected because the IB and plasmapause observations are made at different UT and MLT times. In addition, we found that a few cases showing anomalous energy dispersion ( 3 of 50 conjunctions, in which the $30 \mathrm{keV}$ IB occurred at lower latitude compared to the $80 \mathrm{keV}$ proton IB) lie within the plasmasphere. Anomalous energy dispersion is inconsistent with the CSS scenario and indicates that the IBs are formed by another scattering mechanism. As discussed in the next section, these observations may provide an indication of the wave-related scattering mechanism.

\section{Discussion}

Using excellent coverage provided by the cluster of three THEMIS spacecraft, we investigated the values of the $K$ parameter in the equatorial roots of the proton isotropy boundaries statistically and compared them with the critical value $K_{\text {cr }}=8$ predicted by the current sheet scattering mechanism. 
(a)

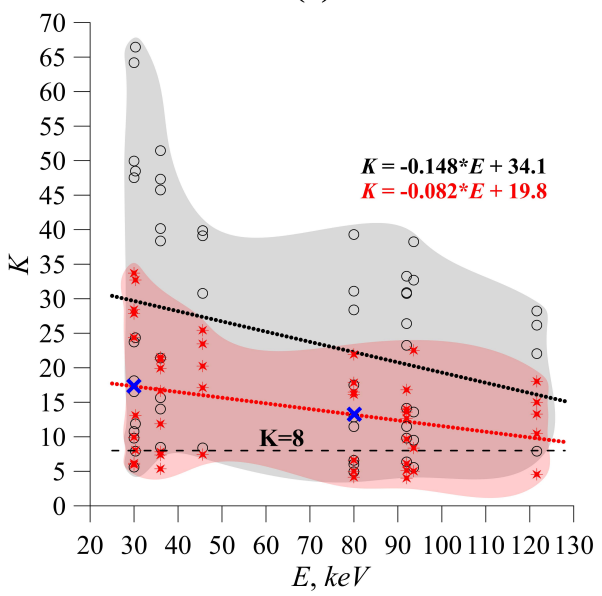

(b)

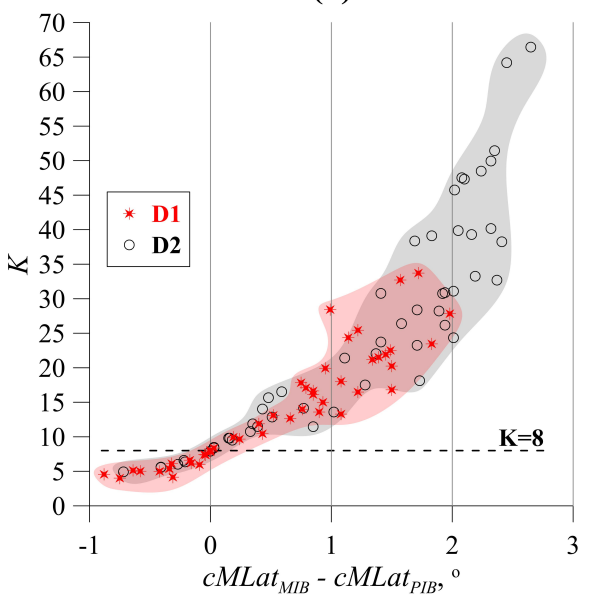

Figure 5. $K$ distribution depending on (a) degradation-corrected lower energy of the proton channel and (b) latitudinal distance between PIB and equatorial point MOB where $K=8$ (see Fig. 2a for the illustration).

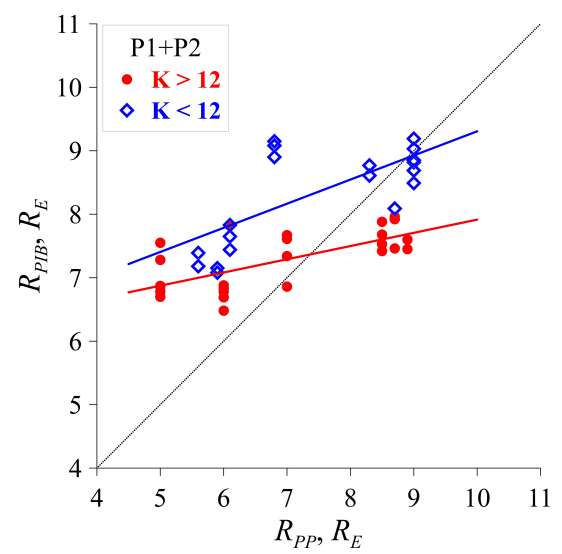

Figure 6. Comparison of mapped IB radial distances (RPIB) with the concurrent plasmapause distances (RPP) for the groups with small $K$ (blue) and large $K$ (red) values. Only the group D1, with the most accurate models, has been analyzed in this plot.

Most events occurred during quiet conditions; special care was taken to avoid the potential cases of inaccurate mapping. Nevertheless, the $K_{\mathrm{PIB}}$ values, obtained by the IB mapping onto the neutral sheet along the model magnetic field line, show a wide spread (Fig. 4). The $K_{\text {PIB }}$ values far exceed the critical value 8 in the majority of events, implying that the PIB is located closer to the Earth compared to predictions of the CSS mechanism. The origin of this large spread and earthward PIB shift may have physical reasons and/or be partly due to errors in the magnetic field model and, consequently, in the field line mapping.

Comparison of $K$ distributions for the groups with more accurate (or less accurate) adaptive models show significant differences between them (Fig. 5), indicating that the accuracy of the modeling and mapping is a strong factor affecting the $K$ values. From this, we got indications that the mapping accuracy mainly depends on the coverage (closest distance of observing spacecraft to the projected field line) and that it degrades notably if this distance exceeds $\sim 2 \mathrm{Re}$ in the inner magnetosphere modeling study. This is a critical and very restrictive requirement for this kind of studies. For the best accuracy group, the $K_{\text {PIB }}$ values do not exceed 35 ; all these points stay within 2 Re distance from the $K=8$ boundary in the equatorial magnetosphere.

In this best-coverage group, a significant number of cases still have $K_{\text {PIB }}$ values exceeding $2 K_{\mathrm{cr}}=16$, suggesting that other mechanisms (different from the current sheet scattering) may be responsible for the strong pitch-angle diffusion in the flux tube adjacent to the isotropy boundary. A plausible mechanism is the resonant interaction with the EMIC waves. Recently, Liang et al. (2014) invoked this mechanism for the explanation of inverse IB energy dispersion for lowenergy proton components $\left(E_{\mathrm{p}}<20 \mathrm{keV}\right)$; they also directly observed EMIC waves and demonstrated the consistency between the observations and the theory predictions. In the statistical survey of proton IB dispersion using NOAA observations, Sergeev et al. (2015) also identified a few morphological features (such as frequent occurrence of coincident IBs in $\mathrm{P} 1$ and $\mathrm{P} 2$ energy channels, frequent multiple structure of the precipitated to trapped flux ratio near the IB location, or observations of newly emerging isotropic precipitation equatorward of the previous IB) which are inconsistent with a simple CSS-based model but can be explained in terms of a waveparticle interaction mechanism.

Three of our results are consistent with the wave mechanism. The first is that large $K_{\mathrm{PIB}}$, far exceeding the $K_{\mathrm{cr}}=8$ value, is observed in the majority of our events, pointing to some wave-interaction-based, strong diffusion mechanism operating in the inner magnetosphere, in the region being $1-2 \mathrm{Re}$ closer to the Earth compared to the CSS-scattering 
$\left(K_{\mathrm{cr}}=8\right)$ boundary. Second, the EMIC wave generation is known to be sensitive to the cold plasma density whose increase leads to the decrease in the minimum resonant energy. The observed tendency of the projected isotropy boundary to occur near the plasmapause or even inside the plasmasphere for large $K$ events (Fig. 6) is, therefore, quite natural for the resonant wave mechanism of strong pitch-angle diffusion. The third fact, that average $K$ values are larger for $30 \mathrm{keV}$ than for $80 \mathrm{keV}$ protons is also consistent with theoretical predictions; see, e.g., Fig. 3 and the discussion in Liang et al. (2014). Direct observations of the intense EMIC waves near the foot of the large $K$ isotropy boundary event are an important future task for observational studies.

For the sake of completeness, one has to consider another possibility of explaining large $K$ values, which does not rule out the main role of the current sheet scattering mechanism. In fact, the $K_{\mathrm{cr}} \approx 8$ criterion was obtained by the tracing of charged-particle trajectories in the simple model field, including the parabolic 1-d current sheet (e.g., Sergeev and Tsyganenko, 1982; Delcourt et al., 1996) or the superposition of this 1-d sheet on the dipole field (Sergeev and Malkov, 1988). Changing the current distribution in the model may alter the pitch-angle scattering results and modify the critical $K$ value (or make it entirely unusable). For example, the addition of a significant $B_{y}$ component will make the scattering amplitude different for particles moving toward the Northern or Southern Hemisphere (Delcourt et al., 2000). Another example is the bifurcated thin current sheet, in which the $K$ value estimated in the neutral sheet is actually meaningless (Delcourt et al., 2004). One more possibility is a case of radially thin current structures, in which the term $\mathrm{d} B_{z} / \mathrm{d} r$ may play the main role (instead of $\mathrm{d} B_{x} / \mathrm{d} z$ ) in determining the scattering amplitude. Such localized current filaments and associated large radial $B$ gradients may be formed in the region of interest $(6-8 \mathrm{Re})$ by the enhanced convection during the substorm growth phase or during the flow burst interaction with the plasma sheet as has been shown numerically in self-consistent Rice Convection Model simulations (Yang et al., 2013, 2014). Previously, thin plasma boundaries (of $30 \mathrm{keV}$ proton gyroradius scale) have been inferred based on Cluster observations and interpreted as a result of flow burst interaction with the inner magnetosphere (Sergeev et al., 2003). These questions provide an interesting topic for a future study. Unfortunately, we have very few opportunities to diagnose such unusual current distributions based on spacecraft observations.

Acknowledgements. This study was supported by the Russian Foundation of Basic Research grant 13-05-00132. The NOAAPOES particle data were provided from NOAA National Geophysical Data Center, http://ngdc.noaa.gov. We thank K. H. Glassmeier, U. Auster and W. Baumjohann for the use of FGM data provided under the lead of the Technical University of Braunschweig and with financial support through the German Ministry for Economy and Technology and the German Center for Aviation and Space
(DLR) under contract 50 OC 0302 . The work by V. Angelopoulos was partly supported by THEMIS contract NAS5-02099. The work by N. Y. Ganushkina was partly supported by the Academy of Finland and by NASA Award NNX14AF34G. We thank M. Holeva for help in manuscript preparation.

The topical editor C. Owen thanks F. Soraas and one anonymous referee for help in evaluating this paper.

\section{References}

Asikainen, T., Mursula, K., and Maliniemi, V.: Correction of detector noise and recalibration of NOAA/MEPED energetic proton fluxes, J. Geophys. Res., 117, A09204, doi:10.1029/2012JA017593, 2012.

Delcourt, D. C., Sauvaud, J.-A., Martin Jr., R. F., and Moore, T. E.: On the nonadiabatic precipitation of ions from the near-Earth plasmasheet, J. Geophys. Res., 101, 17409-17418, 1996.

Delcourt, D. C., Zelenyi, L. M., and Sauvaud, J.-A.: Magnetic moment scattering in a field reversal with nonzero BY component, J. Geophys. Res., 105, 349-359, doi:10.1029/1999JA900451, 2000.

Delcourt, D. C., Malova, H. V., and Zelenyi, L. M.: Dynamics of charged particles in bifurcated current sheets: The $k \approx 1$ regime, J. Geophys. Res., 109, A01222, doi:10.1029/2003JA010167, 2004.

Donovan, E. F., Jackel, B. J., Voronkov, I., Sotirelis, T., Creutzberg, F., and Nicholson, N. A.: Ground-based optical determination of the b2iboundary: A basis for an optical MT-index, J. Geophys. Res., 108, 1115, doi:10.1029/2001JA009198, 2003.

Evans, D. S. and Greer, M. S.: Polar orbiting environmental satellite space environment monitor-2: Instrument descriptions andarchive data documentation, NOAA Tech. Memo. 1.4, Space Environ. Lab., Boulder, Colo, 2004.

Ganushkina, N. Yu., Pulkkinen, T. I., Kubyshkina, M. V., Sergeev, V. A., Lvova, E. A., Yahnina, T. A., Yahnin, A. G., and Fritz, T.: Proton isotropy boundaries as measured on mid- and low-altitude satellites, Ann. Geophys., 23, 1839-1847, doi:10.5194/angeo23-1839-2005, 2005.

Kubyshkina M., Sergeev, V., Tsyganenko, N., Angelopoulos, V., Runov, A., Singer, H., Glassmeier, K. H., Auster, H. U., and Baumjohann, W.: Toward adapted time-dependent magnetospheric models: A simple approach based on tuning the standard model, J. Geophys. Res., 114, A00C21, doi:10.1029/2008JA013547, 2009.

Kubyshkina, M., Sergeev, V., Tsyganenko, N., Angelopoulos, V., Runov, A., Donovan, E., Singer, H., Auster, U., and Baumjohann, W.: Time-dependent magnetospheric configuration and breakup mapping during a substorm, J. Geophys. Res., 116, A00I27, doi:10.1029/2010JA015882, 2011.

Liang, J., Donovan, E., Ni, B., Yue, Jiang, C. F., and Angelopoulos, V.: Onan energy-latitude dispersion pattern ofion precipitation potentially associated with magnetospheric EMIC waves,J. Geophys. Res.-Space, 119, 8137-8160, doi:10.1002/2014JA020226, 2014.

Lvova, E. A., Sergeev, V. A., and Bagautdinova, G. R.: Statistical study of the proton isotropy boundary, Ann. Geophys., 23, 13111316, doi:10.5194/angeo-23-1311-2005, 2005. 
McCollough, J. P., Gannon, J. L., Baker, D. N., and Gehmeyr, M.: A statistical comparison of commonly used external magnetic field models, Space Weather, 6, S10001, doi:10.1029/2008SW000391, 2008.

Meurant, M., Gérard, J.-C., Blockx, C., Spanswick, E., Donovan, E. F., Hubert, B., Coumans, V., and Connors, M.: EL - a possible indicator to monitor the magnetic field stretching at global scale during substorm expansive phase: Statistical study,J. Geophys. Res., 112, A05222, doi:10.1029/2006JA012126, 2007.

Nishimura, Y., Bortnik, J., Li, W., Thorne, R. M., Lyons, L. R., Angelopoulos,V., Mende,S. B., Bonnell, J., Le Contel, O., Cully, C., Ergun,R., and Auster, U.: Estimation of magnetic field mapping accuracy using the pulsating aurora?chorus connection, Geophys. Res. Lett., 38, L14110, doi:10.1029/2011GL048281, 2001.

Petrukovich, A. A., Artemyev, A. V., Malova, H. V., Popov, V. Y., Nakamura, R., and Zelenyi, L. M.: Embedded current sheets in the Earth's magnetotail, J. Geophys. Res., 116, A00I25, doi:10.1029/2010JA015749, 2011.

Sadanger, M. I., Ødegaard, L.-K. G., Tyssøy, H. N., Stadsnes, J., Søraas, F., Oksavik, K., and Aarsnes, K.: In flight calibration of NOAA POES proton detectors - derivation of the MEPED correction factors, J. Geophys. Res. Space, 120, doi:10.1002/2015JA021388, 2015

Sergeev, V. A. and Gvozdevsky, B. B.: MT-index - a possible new index to characterize the magnetic configuration of magnetotail, Ann. Geophys., 13, 1093-1103, doi:10.1007/s00585-995-10939, 1995.

Sergeev, V. A. and Malkov, M. V.: Diagnostic of the magnetic configuration of the plasma sheet from measurements of energeticelectrons above the ionosphere, Geomagn. Aeron., 28, 649-653, 1988.

Sergeev, V. A. and Tsyganenko, N. A.: Energetic particle losses and trapping boundaries as deduced from calculations with a realisticmagnetic field model, Planet. Space Sci., 10, 999-1006, 1982.

Sergeev, V. A., Sazhina, E. M., Tsyganenko, N. A., Lunbland, J. A., and Soraas, F.: Pitch angle scattering of energetic protons in themagnetotail current sheet as the dominant source of their isotropic precipitation into the nightside ionosphere, Planet. Space Sci., 31, 1147-1158, 1983.
Sergeev, V. A., Sauvaud, J.-A., Reme, H., Balogh, A., Daly, P., Zong, Q.-G., Angelopoulos, V., Andre, M., and Vaivads, A.: Sharp boundary between the inner magnetosphere and active outer plasma sheet, Geophys. Res. Lett., 30, 1799, doi:10.1029/2003GL017095, 2003.

Sergeev, V. A., Chernyaeva, S. A., Apatenkov, S. V., Ganushkina, N. Y., and Dubyagin, S. V.: Energy-latitude dispersion patterns near the isotropy boundaries of energetic protons, Ann. Geophys., 33, 1059-1070, doi:10.5194/angeo-33-1059-2015, 2015.

Shevchenko, I. G., Sergeev, V., Kubyshkina, M., Angelopoulos, V., Glassmeier, K. H., and Singer, H. J.: Estimation of magnetosphere-ionosphere mapping accuracy using isotropy boundary and THEMIS observations, J. Geophys. Res., 115, A11206, doi:10.1029/2010JA015354, 2010.

Tsyganenko, N. A.: Modeling the Earth's magnetospheric magnetic field confined within a realistic magnetopause, J. Geophys. Res. 100, 5599-5612, doi:10.1029/94JA03193, 1995.

Wang, C.-P., Zaharia, S. G., Lyons, L. R., and Angelopoulos, V.: Spatial distributions of ion pitchangle anisotropy in the nearEarth magnetosphere and tail plasma sheet, J. Geophys. Res.Space, 118, 244-255, doi:10.1029/2012JA018275, 2012.

Yahnin, A. G. and Yahnina, T. A.: Energetic proton precipitation related to ion-cyclotron waves, J. Atmos. Terr. Phys., 69, 16901706, 2007.

Yang, J., Wolf, R. A., Toffoletto, F. R., and Sazykin, S.: RCM-E simulation of substorm growth phase arc associatedwith largescale adiabatic convection, Geophys. Res. Lett., 40, 6017-6022, doi:10.1002/2013GL058253, 2013.

Yang, J., Toffoletto, F. R., and Wolf, R. A.: RCM-E simulation of a thin arc preceded by a north-south-aligned auroral streamer, Geophys. Res. Lett., 41, 2695-2701, doi:10.1002/2014GL059840, 2014.

Yue, C., Wang, C.-P., Donovan, E. F., Zaharia, S. G., Lyons, L., Liang, J., and Henderson, M.: Current sheet scattering and ion isotropicboundary under 3D empirical force-balanced magnetic field, J. Geophys. Res.-Space, 119, 8202-8211, doi:10.1002/ 2014JA020172, 2014. 